\title{
Acellular micronized extracellular matrix and occlusive dressings for open fingertip injuries
}

\author{
Stephanie E. Dreifuss ${ }^{1}$, Ronit Wollstein ${ }^{1,2}$, Stephen F. Badylak ${ }^{1}$, Peter J. Rubin ${ }^{1}$ \\ ${ }^{1}$ Department of Plastic and Reconstructive Surgery, University of Pittsburgh, Pittsburgh, PA 15261, USA. \\ ${ }^{2}$ Department of Orthopedic Surgery, Technion Medical School, Haifa 3200003, Israel.
}

Address for correspondence: Prof. Ronit Wollstein, Department of Plastic and Reconstructive Surgery, University of Pittsburgh, Pittsburgh, PA 15261, USA. E-mail: ronitwollstein@gmail.com

Sir,

Fingertip amputation injuries compromise the sensation, function, and appearance of the hand. They are often exquisitely painful and take a substantial amount of time to heal, causing significant functional disability. Conservative management of these injuries may result in decreased range of motion, hypo- or hyperesthesia, loss of pulp contour, scarring, and other long-term sequelae. ${ }^{[1,2]}$ In addition, they may prolong patient discomfort with dressing changes, hindering adherence with wound therapy regimens, especially in the presence of multiple finger injuries.

Both acellular and cellular bioscaffolds have been employed in conjunction with wound dressing techniques. These biologically active materials supply extracellular matrix proteins, deliver growth factors, and recruit differentiated cells to the wound site. ${ }^{[3,4]}$ Furthermore, they aim to address the issue of pain associated with debriding dressings. The efficacy of cellular materials is limited by their reduced shelf life, risk of the immune response, increased regulation and high cost. Acellular materials, however, do not face these limitations.

We present a series of 8 patients $(6$ males and 2 females, average age: $40.8 \pm 18.8$ years) with fingertip amputations treated with Particulate Extracellular Matrix, or P-ECM (ACell Inc., Columbia, MD), an acellular, sterile, porcine-derived, naturally occurring, lyophilized and extracellular matrix material. There were 11 fingers treated. Injuries in these patients involved fingertip pulp

\begin{tabular}{|l|l|}
\hline \multicolumn{2}{|c|}{ Access this article online } \\
\hline Quick Response Code: & Website: \\
\hline & www.parjournal.net \\
\hline & \\
\hline
\end{tabular}

only, pulp and nail bed, or pulp, nail bed, and distal phalanx. All patients in the series were followed until complete wound healing, which occurred within an average of 7 weeks.

The wound care regimen for all patients involved removal of dressings, gentle sterile saline rinse, application of additional P-ECM powder, and redressing with nonadherent petrolatum gauze and cotton gauze fingertip bandage. The P-ECM was not completely removed with each dressing change but allowed to form a crystallized layer over the wound.

All patients rated the comfort of P-ECM treatment as 5 on a 5-point scale, citing a soothing effect upon changing dressings. Ease of use was rated as 4.2 out of 5 for patients who had another individual apply the treatment, while patients commented on the difficulty of independently treating and dressing their own fingertip wounds. Moreover, patients reported satisfaction with the appearance and function of their fingertips in comparison with normal digits. All patients noted a subjective recovery of pressure sensation, the light touch, and temperature sensation, comparable to their uninjured digits. There were no complications associated with the use of P-ECM in our series.

The P-ECM powder has an advantage of being applied every other day. Furthermore, application and maintenance are simple and comfortable for patients.

This is an open access article distributed under the terms of the Creative Commons Attribution-NonCommercial-ShareAlike 3.0 License, which allows others to remix, tweak, and build upon the work non-commercially, as long as the author is credited and the new creations are licensed under the identical terms.

For reprints contact: reprints@medknow.com

How to cite this article: Dreifuss SE, Wollstein R, Badylak SF, Rubin PJ. Acellular micronized extracellular matrix and occlusive dressings for open fingertip injuries. Plast Aesthet Res 2015;2:282-3.

Received: 23-11-2014; Accepted: 03-01-2015 
This is especially true for patients with multiple fingertip amputations in which dressing changes may be a painful ordeal. Like all treatments that allow for wound healing by secondary intention, the P-ECM technique requires that a patient adequately understand the treatment protocol and correctly apply the powder every other day. Therefore, it is important to consider patient adherence and availability of an individual who can aid in treatment application, patient's access to sterile saline and wound dressing materials, and motivation to adhere to treatment regimen.

This series demonstrates that the use of P-ECM is comparable to other treatment options for fingertip amputations. Its use was not compared to healing by secondary intention or any surgical procedures. At this time, there is no clear justification for its use in fingertip amputations, but perhaps it should be kept in our toolbox for the treatment of fingertip injuries, especially when there are multiple fingers involved. Study is necessary to examine the advantages, disadvantages and to define the indications for its use.

\section{Acknowledgment}

P-ECM powder was provided by ACell, Inc., Columbia, MD.

Financial support and sponsorship

Nil.

\section{Conflicts of interest}

There are no conflicts of interest.

\section{REFERENCES}

I. Yeo CJ, Sebastin SJ, Chong AK. Fingertip injuries. Singapore Med J 2010;5।:78-86.

2. Fassler PR. Fingertip Injuries: evaluation and treatment. J Am Acad Orthop Surg 1996;4:84-92.

3. Rivera AE, Spencer JM. Clinical aspects of full-thickness wound healing. Clin Dermatol 2007;25:39-48.

4. Lazic $T$, Falanga $\mathrm{V}$. Bioengineered skin constructs and their use in wound healing. Plast Reconstr Surg 20 I I; 27 Suppl I:S75-90. 